\title{
Hemolytic Activity of pH-Responsive Polymer-Streptavidin Bioconjugates $^{\dagger}$
}

\author{
Chantal A. Lackey, ${ }^{\ddagger}$ Niren Murthy, ${ }^{\ddagger}$ Oliver W. Press, ${ }^{\S}$ David A. Tirrell," Allan S. Hoffman, ${ }^{*, \perp}$ and \\ Patrick S. Stayton*,\#
}

Department of Bioengineering and Department of Medicine, University of Washington, Seattle, WA 98195, and Department of Chemical Eng'g, Calif. Institute of Technology, Pasadena CA. Received September 11, 1998; Revised Manuscript Received March 11, 1999

\begin{abstract}
Drug delivery systems that increase the rate and/or quantity of drug release to the cytoplasm are needed to enhance cytosol ic delivery and to circumvent nonproductive cell trafficking routes. We have previously demonstrated that poly(2-ethylacrylic acid) (PEAAc) has $\mathrm{pH}$-dependent hemolytic properties, and more recently, we have found that poly(2-propylacrylic acid) (PPAAc) displays even greater $\mathrm{pH}$ responsive hemolytic activity than PEAAc at the acidic pHs of the early endosome. Thus, these polymers could potentially serve as endosomal releasing agents in immunotoxin therapies. In this paper, we have investigated whether the $\mathrm{pH}$-dependent membrane disruptive activity of PPAAc is retained after binding to a protein. We did this by measuring the hemolytic activity of PPAAc-streptavidin model complexes with different protein to polymer stoichiometries. Biotin was conjugated to amine-terminated PPAAc, which was subsequently bound to streptavidin by biotin complexation. The ability of these samples to disrupt red blood cell membranes was investigated for a range of polymer concentrations, a range of $\mathrm{pH}$ values, and two polymer-to-streptavidin ratios of 3:1 and 1:1. The results demonstrate that (a) the PPAAC-streptavidin complex retains the ability to lyse the RBC lipid bilayers at low $\mathrm{pHs}$, such as those existing in endosomes, and (b) the hemolytic ability of the PPAAc-streptavidin complex is similar to that of the free PPAAC.
\end{abstract}

\section{INTRODUCTION}

There are now many biomolecular drugs in development for the treatment of a wide variety of medical ailments. These therapeutics include DNA for gene therapies, oligonucleotides for antisense therapies, and protein or peptide drugs and toxins for disease (e.g., cancer) therapies. A significant problem with the development of many DNA and protein therapies is the delivery of the biomolecule to the proper intracellular site. The efficacy of DNA and protein therapeutics is often limited by cellular trafficking processes that shunt the agents to undesirable compartments or recycle them out of the cell (1). Both DNA and protein therapeutics rely on complicated trafficking routes initiated with receptormediated endocytosis followed by transport through endosomal, endoplasmic reticulum, and/or trans-Golgi compartments. The efficiency of delivery across these compartments to the cytosol, a necessary step in gene therapy and many protein therapies, is often the limiting factor in treatment efficacy.

When drugs and their complexes or conjugates with carriers are endocytosed, they are often rapidly trafficked

† C.A.L. was awarded the 1998 Controlled Release SocietyCygnus Graduate Student Award for Outstanding Work in Drug Delivery for this work.

* To whom correspondence should be addressed.

‡ Department of Bioengineering.

$\S$ Department of Medicine.

" Department of Chemical Eng'g.

$\perp$ Department of Bioengineering, Box 352255. Phone: 206543-9423. Fax: 206-543-6124. E-mail: hoffman@biong.washington. edu.

\#Department of Bioengineering, Box 352125. Phone: 206685-8148. Fax: 206-685-8256. E-mail: stayton@biœeng.washington.edu. from early endosomes to late endosomes, and eventually to lysosomes where they are degraded (2). I nternalized drugs are not normally rel eased into the cytoplasm of the cell. An important example is the Immunotoxin family, which exploits the specificity of monoclonal antibodies for delivery of toxins to specific cellular and tissue targets. The most commonly utilized toxins are ricin, a plant toxin from the seeds of Ricinus communis, and the bacterial toxins Diphtheria toxin and Pseudomonas exotoxin A (3). The efficacy of immunotoxins has been shown to be dependent on the specific monoclonal antibody used (3). Antibodies internalized by endocytosis are often trafficked to lysosomes where they are degraded (4), unlike the native toxin which is translocated into the cytosol (5). Previous attempts to circumnavigate this trafficking limitation include the modification of ricin A chain by the addition of endoplasmic reticulum and Gol gi retention sequences (6), the use of endosome-destabilizing peptides (7), and $\mathrm{pH}$-triggered bispecific antibodies (8, 9).

We have been investigating $\mathrm{pH}$-sensitive or "intelligent" polymers that are capable of lysing red blood cells at $\mathrm{pH}$ values observed in endosomes, while being nondisruptive at a physiologic $\mathrm{pH}$ of 7.4. These are based on $\alpha$-alkyl acrylic acids. One example is poly(2-ethylacrylic acid) (PEAAC), ${ }^{1}$ which has been shown to have $\mathrm{pH}$ dependent membrane-disruptive properties in liposomes (10). A pH-triggered conformational change occurs at $\mathrm{pH}$

1 PEAAc, poly(2-ethylacrylic acid); PPAAc, poly(2-propylacrylic acid); PPAAc- $\mathrm{NH}_{2}$, amine-terminated PPAAc; PPAAc-biotin, biotinylated PPAAC; RBC, red blood cell; GPC, gel permeation chromatography; NHS-LC-biotin, Sulfosuccinimidyl-6-(biotinamido)-6-hexanoate; HABA, hydroxyazobenzene-2-carboxylic acid; SDS-PAGE, sodium dodecyl sulfate-polyacrylamide gel electrophoresis. 
6.3 and below $(11,12)$. At these lower $\mathrm{pH}$ conditions, PEAAc appears to permeabilize phosphatidylchol ine (PC) membranes, disrupt the lamellar structure of lipid bilayers, and form mixed micelles composed of polymer and lipid. This process has a high efficiency, and the disruption of PC membranes in liposomes is general, as PEAAC disrupts distearoyl, dipalmitoyl, dimyristoyl, dilauroyl, and dioleoyl PC at low pH (10). These observations suggested that these polymers may be useful as a new class of drug delivery formulation components that enhance delivery of biomolecules to the cytoplasm.

We have recently shown that PEAAC and PPAAC efficiently induce red blood cell (RBC) hemolysis at lower $\mathrm{pH}$, such as those existing in endosomes $(13,14)$. To be effective in assisting endosomal release of therapeutics, PEAAC and PPAAC must retain their hemolytic activity when conjugated to a protein. In this paper, we have prepared complexes of PPAAc and streptavidin at stoichiometries of 1:1 and 3:1 polymer to protein. This serves as a model to test the ability of the membrane-disruptive polymer when linked to a protein to disrupt the lipid bilayers of RBC membranes at the pHs existing in endosomes.

\section{EXPERIMENTAL PROCEDURES}

Polymer Preparation. Amine-terminated PPAAC (PPAAC $-\mathrm{NH}_{2}$ ) was synthesized using establ ished chaintransfer free-radical polymerization techniques. The propyl acrylic acid monomer was mixed with 1 mol \% initiator (azobisisobutyronitrile) and 4 mol \% chaintransfer agent (cysteamine). The mixture was degassed by first freezing in liquid nitrogen, and subsequently placed under vacuum and thawed. This was repeated three times. Polymerization was carried out at $60^{\circ} \mathrm{C}$. Two different PPAAc preparations were used in subsequent experiments. The product of the first PPAAc polymerization reaction was fractionated by preparative gel permeation chromatography (GPC), while ether precipitation was used to remove small polymer chains from the second PPAAc polymerization products.

Biotinylation of PPAAc. PPAAC $-\mathrm{NH}_{2}$ was biotinylated using sulfosuccinimidyl-6-(biotinamido)-6-hexanoate (EZ-Link sulfo-NHS-LC-biotin, Pierce). PPAAC- $\mathrm{NH}_{2}$ was dissolved in $0.1 \mathrm{M}$ sodium phosphate and $0.15 \mathrm{M}$ $\mathrm{NaCl}, \mathrm{pH}$ 7.4. The NHS-LC-biotin was dissolved in water at a concentration of $20 \mathrm{mg} / \mathrm{mL}$ and added to each of the polymers at a 20 times molar excess. This was reacted for $2 \mathrm{~h}$ at room temperature. The biotinylation was repeated using a 15 times molar excess of biotin to maximize the yield of biotinylated polymer molecules. The samples were purified using a PD-10 desalting col umn (Pharmacia) in order to ensure that all the free biotin was removed. Absorbance at $220 \mathrm{~nm}$ was used to detect which fractions contained polymer, and these fractions were subsequently pooled.

Determination of Concentration of PPAAC-Biotin. To determine the concentration of the biotinylated PPAAC (PPAAC-biotin), the neutravidin-HABA dye assay was used. A total of $1.5 \mathrm{mg}$ of neutravidin (Molecular Probes) was dissolved in $3 \mathrm{~mL}$ of $0.1 \mathrm{M}$ sodium phosphate and $0.15 \mathrm{M} \mathrm{NaCl}, \mathrm{pH}$ 7.4. 4'-Hydroxyazobenzene-2carboxylic acid (HABA) (Sigma) was dissolved in $10 \mathrm{mM}$ $\mathrm{NaOH}$ at a concentration of $2.42 \mathrm{mg} / \mathrm{mL}(10 \mathrm{mM})$, and $75 \mu \mathrm{L}$ was added to the protein. D-Biotin (Sigma) was dissolved in $0.1 \mathrm{M}$ sodium phosphate and $0.15 \mathrm{M} \mathrm{NaCl}$, $\mathrm{pH} 7.4$, at a concentration of $0.5 \mathrm{mM}$. This solution was added in $10 \mu \mathrm{L}$ aliquots to the protein-HABA complex, while the absorbance at $500 \mathrm{~nm}$ was measured after each addition. By plotting the absorbance at $500 \mathrm{~nm}$ versus the amount of biotin added, the calibration curve was constructed. In parallel, incremental amounts of PPAACbiotin were added to the neutravidin and HABA solution, and the absorbance at $500 \mathrm{~nm}$ was measured. The slope of the curve generated was compared to the calibration curve in order to determine the concentration.

Hemolysis Assay. The $\mathrm{pH}$-dependent membranedisruptive activity of the PPAAc was measured using a red blood cell (RBC) hemolysis assay. RBCs were harvested by centrifuging whole blood for $4 \mathrm{~min}$. They were washed three times with $100 \mathrm{mM}$ dibasic sodium phosphate at the desired $\mathrm{pH}$, and resuspended in the same buffer to yield the initial volume. They were diluted 10 times in the same buffer, and $200 \mu \mathrm{L}$ of this suspension was used for each tube. This yields $10^{8} \mathrm{RBCs}$ per tube. Each tube contained $800 \mu \mathrm{L}$ of buffer, $200 \mu \mathrm{L}$ of the RBC suspension, and the polymer. Each sample was done in triplicate, and was then repeated to verify reproducibility. The tubes were incubated for an hour and a half in a 37 ${ }^{\circ} \mathrm{C}$ incubator. They were spun for $5 \mathrm{~min}$ at full speed in the mi crocentifuge. Lysis was determined by measuring the absorbance of the supernatant at $541 \mathrm{~nm}$, reflecting the amount of hemogl obin which had been released into the supernatant. Percent hemolysis was calculated assuming $100 \%$ lysis to be measured by the hemoglobin released by the red blood cells in water; controls of RBCs in buffer with no polymer or in buffer with added streptavidin were also run.

\section{RESULTS}

Polymer Preparation and Properties. To investigate the importance of polymer polydispersity, the PPAAC was fractionated by preparative GPC. The resulting polymer mixture displayed an average molecular mass that is equivalent to a PEG standard of $20 \mathrm{kDa}$. We found that the hemolytic activity of the polymer was not strongly dependent on the degree of polydispersity, as ether-precipitated PPAAC with higher polydispersities demonstrated the same hemolytic activity as fractionated PPAAC.

Biotinylation of PPAAc. The neutravidin-HABA assay was used to determine the fraction of PPAAc that was biotinylated. The results demonstrate that no detectable quantity of the polymer remains unbiotinylated. In addition, if unbiotinylated PPAAc remained in the mixture, we would expect that the concentration dependence of the hemolysis assay (below) would be shifted to reflect the higher concentration added by the nonderivatized polymer. The biotinylation of PPAAC was also verified with an SDS-PAGE gel shift assay (Figure 2). Samples of streptavidin al one, a physical mixture of streptavidin and PPAAC $-\mathrm{NH}_{2}$, and a complex of streptavidin and PPAAc-biotin were analyzed. The biotin-streptavidin affinity was exploited to create the streptavidin-biotinylated polymer complex as diagrammed in Figure 3. Because the polymer is negatively charged at neutral $\mathrm{pH}$, the streptavidin-bi otinylated PPAAc complex migrates further in the gel. The physical mixture of streptavidin and PPAAC- $\mathrm{NH}_{2}$ does not display a shift in electrophoretic mobility, indicating that the interaction between streptavidin and PPAAc is specifically mediated by biotin.

Hemolysis Assay. The abilities of five different samples to hydrolyze RBCs were compared. Test samples included amine-terminated PPAAc; biotinylated PPAAc; a physical mixture of streptavidin + amine-terminated PPAAC; a streptavidin-biotinylated PPAAc complex; streptavidin alone; and RBCs alone. The abilities of these samples to 


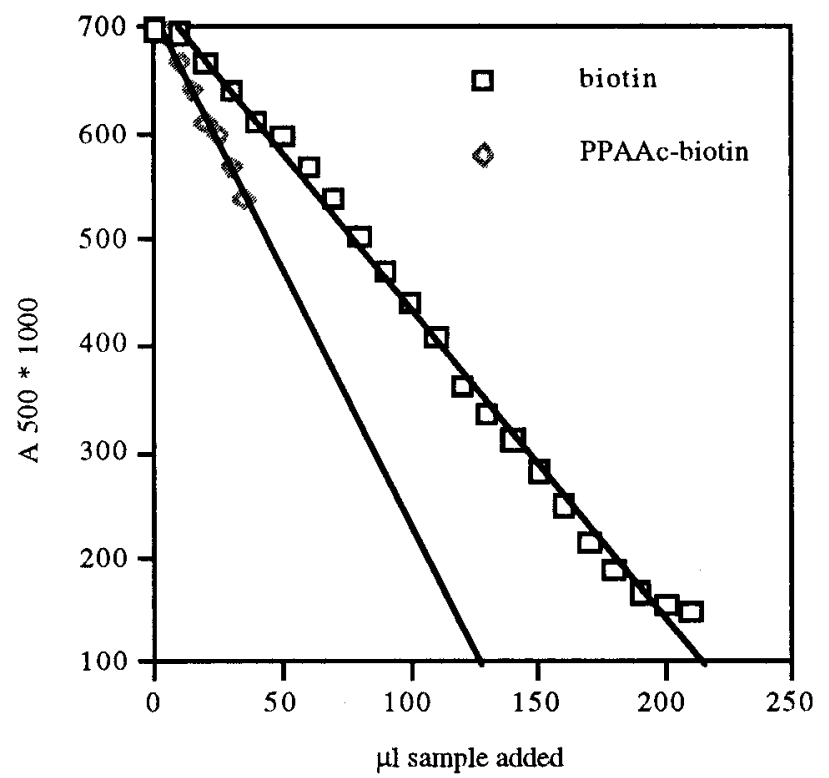

Figure 1. Determination of PPAAc-biotin concentration using the neutravidin/HABA assay.

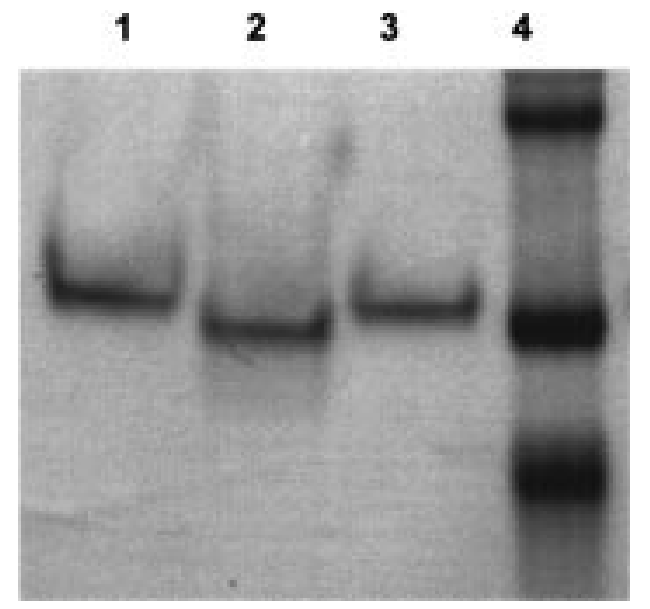

Figure 2. (1) PPAAC- $\mathrm{NH}_{2}+$ Streptavidin, (2) PPAAc-biotin/ Streptavidin, (3) Streptavidin, (4) MW marker.

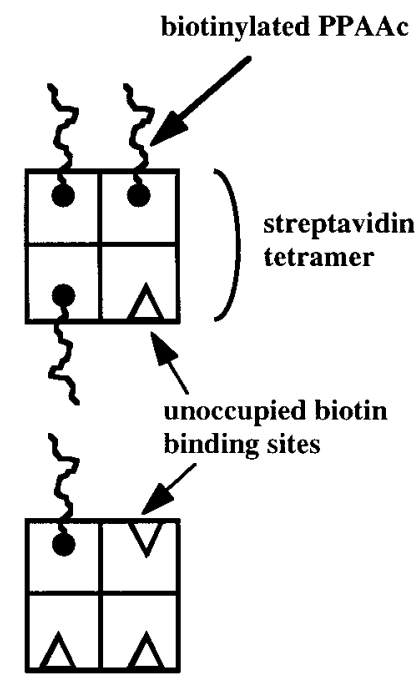

Figure 3. Schematic of the streptavidin-polymer model system with the 3:1 and 1:1 polymer:protein ratios.

disrupt RBC membranes was investigated for a range of polymer concentrations, a range of $\mathrm{pH}$ values, and for two polymer-to-streptavidin ratios of 3:1 and 1:1. Figure 4 shows the effect of concentration of PPAAc on hemolysis
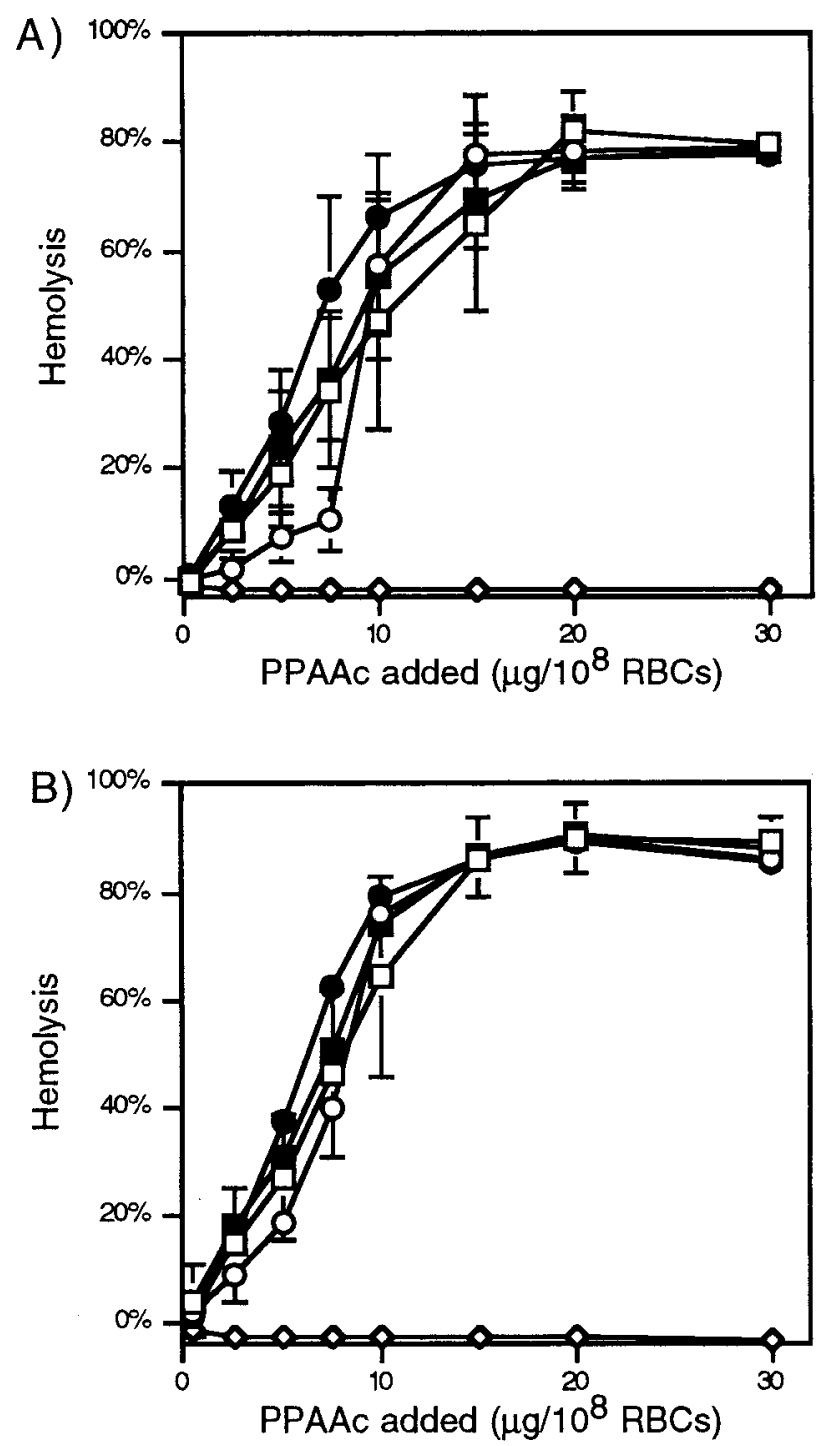

Figure 4. Concentration dependence of hemolytic activity for PPAAc- $\mathrm{NH}_{2}$ (open squares), PPAAc-biotin (open circles), streptavidin + PPAAc- $\mathrm{NH}_{2}$ physical mixture (closed squares), streptavidin/PPAAc-biotin complex (closed circles), and streptavidin alone (diamonds), with a polymer:protein stoichiometry of 3:1 (A) and 1:1 (B).

at $\mathrm{pH} 5$ for a polymer-to-protein ratio of 3:1 (Figure 4A) and 1:1 (Figure 4B). All the samples containing PPAAC display equivalent hemolytic activity, as illustrated by the superimposable curves of all derivatives of PPAAC, which are normalized to the weight of PPAAc in each sample. The maximum hemolytic capability is reached at roughly $15 \mu \mathrm{g}$ of PPAAC per $10^{8}$ RBCS. Figure 5 displays the \% hemolysis versus $\mathrm{pH}$ for PPAAC at a concentration of $15 \mu \mathrm{g}$ of polymer per $10^{8} \mathrm{RBCs}$. Results for polymer-to-protein ratios of 3:1 and 1:1 are presented in Figure 5. A sharp dependence on pH is demonstrated, with no significant hemolytic activity at $\mathrm{pH} 7.4$ and a maximum achieved by $\mathrm{pH}$ 6.3.

\section{DISCUSSION}

The results show that PPAAc hemolyzes red blood cells with the same efficiency whether as a free polymer or complexed via biotin to the protein streptavidin. The hemolysis versus concentration profile is virtually identical for all samples containing PPAAC, indicating that the hemolytic activity of PPAAc is not affected by its linkage to a protein. Hemolysis by PPAAc reaches its maximum 

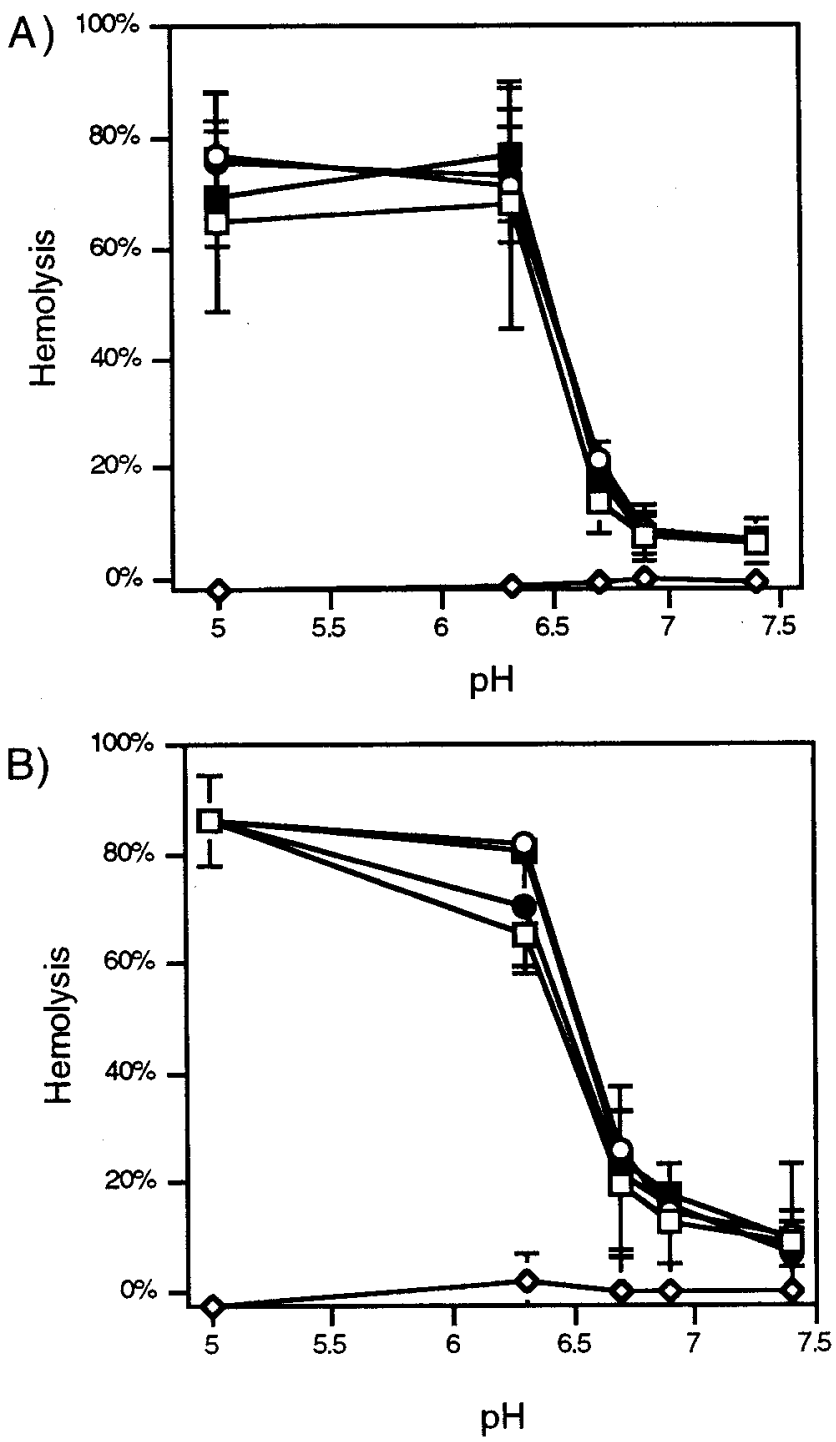

Figure 5. $\mathrm{pH}$ dependence of hemolytic activity for PPAAc$\mathrm{NH}_{2}$ (open squares), PPAAc-biotin (open circles), streptavidin + PPAAc $-\mathrm{NH}_{2}$ physical mixture (closed squares), streptavidin/ PPAAc-biotin complex (closed circles), and streptavidin alone (diamonds), with a polymer:protein stoichiometry of 3:1 (A) and 1:1 (B). All samples with polymer contained $15 \mu \mathrm{g}$ of PPAAC.

at ca. $15 \mu \mathrm{g}$ of polymer $/ 10^{8}$ red blood cells, which corresponds to ca. $4.5 \times 10^{6}$ molecules of PPAAc/red blood cell. Since polymer-to-protein ratios of 3:1 and 1:1 displayed equivalent activity, we can conclude that it is unnecessary to have more than one polymer chain complexed per protein molecule to achieve a highly efficient membrane lytic activity. The hemolysis versus pH curves were also identical for the free PPAAC and for the PPAAC-protein conjugates at both PPAAc:protein ratios. The hemolysis is strongly $\mathrm{pH}$ dependent, with the transition from negligible lysis to maximum lysis occurring as the $\mathrm{pH}$ decreases over a narrow range from $\mathrm{pH} 6.9$ to 6.3. Thus, linkage of PPAAc to the protein does not affect the $\mathrm{pH}$ dependent conformational and physical change of the polymer and its ability to disrupt the RBC membrane.

We have not investigated the mechanism of this action, but Tirrell and co-workers have studied the disruption of liposomes by PEAAc (15). Their surface tensiometry results were consistent with a micellization process driven by hydrophobic interactions of the polymer and the lipid bilayer. Such a mechanism is similar to that seen for amphiphilic, $\alpha$-helix-based polypeptides, but the polymer is unlikely to adopt a secondary structure, implying that the hydrophobic-to-hydrophilic ratio is the most important factor in liposome micellization (15). They also found that the rate of micellization was determined by the rate and extent of PEAAC absorption, which in turn was determined by the lateral compressibility of the membrane (16). In comparison, melittin, the most membrane disruptive peptide known, was less effective on a molar basis (17).

The ability of the PPAAc-streptavidin complexes to disrupt RBC membranes at lowered pHs, such as those present in endosomes, suggests that these unusual $\mathrm{pH}$ responsive polymers represent a new avenue to facilitate endosomal release and to improve the intracellular delivery of protein or DNA therapeutics.

\section{ACKNOWLEDGMENT}

This work was supported by the NIH (NIGMS Grant R01-GM53771-02), the UW Center for Nanotechnology (graduate fellowships to C.A.L. and N.M.), the Office of Technology Transfer at UW, the Washington Technology Center, and the Washington Research Foundation.

\section{LITERATURE CITED}

(1) Press, O. W., Martin, P. . ., Thorpe, P. E., and Vitetta, E. S. (1988) Ricin A-chain containing immunotoxins directed against different epitopes on the CD2 molecule differ in their ability to kill normal and malignant T cells. J . Immunol . 141, 44107.

(2) Mukherjee, S., Ghosh, R. N., and Maxfield, F. R. (1997) Endocytosis. Physiol. Rev. 77, 759-803.

(3) Blakey, D. C. (1992) Drug targeting with monoclonal antibodies. A review. Acta Oncol. 31, 91-7.

(4) Press, O. W., Hansen, J . A., Farr, A., and Martin, P. J . (1988) Endocytosis and degradation of murine anti-human CD3 monoclonal antibodies by normal and malignant T-Iymphocytes. Cancer Res. 48, 2249-57.

(5) Wawrzynczak, E. J . (1991) Systemic immunotoxin therapy of cancer: advances and prospects. Br. J . Cancer 64, 62430

(6) Zhan, J., Stayton, P., and Press, O. W. (1998) Modification of ricin A chain, by addition of endoplasmic reticulum (KDEL) or Golgi (YQRL) retention sequences, enhances its cytotoxicity and translocation. Cancer I mmunol., I mmunother. 46, 5560.

(7) Tolstikov, V. V., Cole, R., Fang, H., and Pincus, S. H. (1997) Influence of endosome-destabilizing peptides on efficacy of anti-HIV immunotoxins. Bioconjugate Chem. 8, 38-43.

(8) Raso, V., Brown, M., McGrath, J ., Liu, S., and Stafford, W. F . (1997) Antibodies capable of releasing diphtheria toxin in response to the low $\mathrm{pH}$ found in endosomes. J. Biol. Chem. $272,27618-22$.

(9) Raso, V., Brown, M., and McGrath, J . (1997) Intracellular targeting with low pH-triggered bispecific antibodies. J . Biol. Chem. 272, 27623-8.

(10) Thomas, J . L., and Tirrell, D. A. (1992) Polyelectrolytesensitized phospholipid vesicles. Acc. Chem. Res. 25, 33642.

(11) Borden, K. A., Eum, K. M., Langley, K. H., and Tirrell, D. A. (1987) On the mechanism of polyelectrolyte-induced structural reorganization in thin molecular films. Macromol ecules 20, 454-6.

(12) Eum, K. M., Langley, K. H., and Tirrell, D. A. (1989) Quasielastic and electrophoretic light scattering studies of the reorganization of dioleoyl phosphatidylcholine veside membranes by poly(2-ethylacrylic acid). Macromolecules 22, 275560.

(13) (a) Murthy, N., Robichaud, J ., Stayton, P. S., Press, O. W., Hoffman, A. S., and Tirrell, D. A. (1998) Design of polymers to increase the efficiency of endosomal release of drugs. Proc. Intl. Symp. Control. Relat. Bioact. Mater. 25, 224-5. (b) Lackey, C. A., Murthy, N., Stayton, P. S., Press, O. W., Hoffman, A. S., and Tirrell, D. A. (1998) Proc. Intl. Symp. Control. Relat. Bioact. Mater. 25, 87-8. 
(14) Murthy, N., Robichaud, J. R., Tirrell, D. A., Stayton, P. S., and Hoffman, A. S. (1998) The design and synthesis of polymers for eukaryotic membrane disruption. J . Control led Rel. (Submitted for publication).

(15) Thomas, J . L., Barton, S. W., and Tirrell, D. A. (1994) Membrane solubilization by a hydrophobic polyelectrolyte: surface activity and membrane binding. Biophysical J . 67, 1101-6.
(16) Thomas, J . L., Devlin, B. P., and Tirrell, D. A. (1996) Kinetics of membrane micellization by the hydrophobic polyelectrolyte poly(2-ethylacrylic acid). Biochim. Biophys. Acta 1278, 73-8.

(17) Dempsey, C. E. (1990) The actions of melittin on membranes. Biochim. Biophys. Acta 1031, 143-61.

BC980109K 